\title{
ENFERMERÍA, CUIDADO Y CIENCIA
}

\author{
Juan Ignacio Rico Becerra
}

Enfermero, Licenciado en Antropología, Licenciado en Geografía e Historia, Doctor en Antropología.

Profesor del Departamento de Antropología Social y Cultural de la Universidad Católica de Murcia (UCAM).

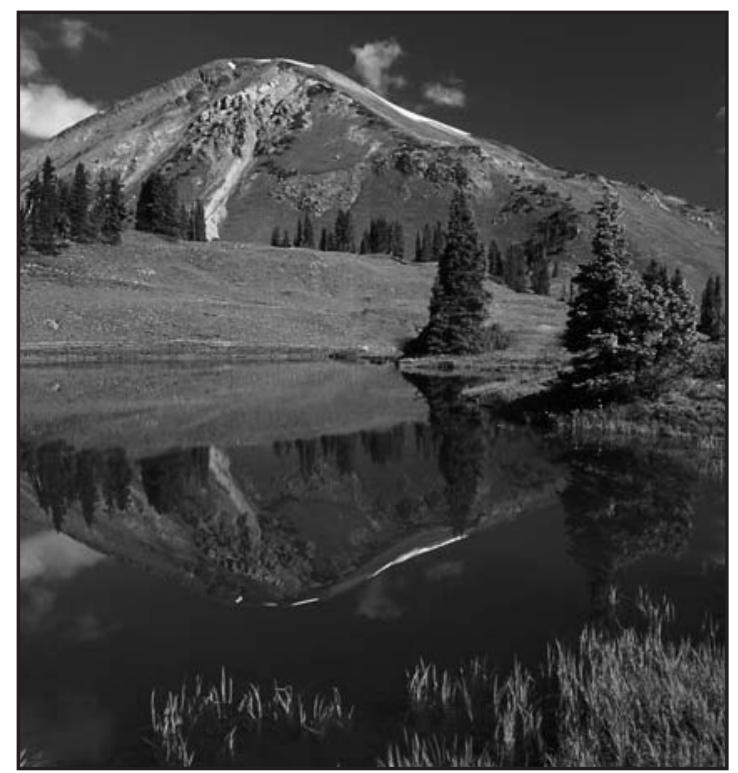

NURSING, CARE AND SCIENCE

\section{SUMMARY}

$\mathrm{N}$ ursing has always trusted in the significance of the human approach within health care. This is a deeply rooted fact that, becomes more evident in the face of the 'biologistic' approach of western, scientific medicine, which at times, can even seem somewhat insulting. In this paper we propose reflection on the theme of health care which we hope will lead to further debate on the whole question. With this in mind we shall cover various issues such as: paradigm, science reflexive modernisation, Eastern, scientific medicine, quantum physics, etc

Key words: Nursing, Cure/care (caring), paradigm, science, science reflexive modernisation, scientific medicine.

\section{ENFERMAGEM, CUIDADO E CIENCIA}

\section{RESUMO}

Enfermagem sempre confiou na importân$\triangle$ cia do cuidado humanizado. E isto no é -1 utopia, sobretudo, nos momentos em que o enfoque biologicista da medicina científico ocidental chegou a ser quase insultante. Nessas linhas proponho uma reflexão na perspectiva do cuidado e que nos leve a abrir um debate mais amplo em que considero que essa questão está inserida. Assim sendo, transitaremos por diversos conceitos, tais como: paradigma, ciência, modernização reflexiva, medicina científica ocidental, física quântica, etc.

Palavras chave: Enfermagem, cuidado, paradigma, ciência, modernização reflexiva, medicina científica ocidental.

\section{RESUMEN}

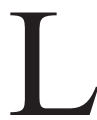

a enfermería siempre ha confiado en la importancia del cuidado humanizado. Y ello no es baladí, sobre todo, en los momentos donde el enfoque biologicista de la medicina científico-occidental ha llegado a ser casi insultante. En estas líneas propongo una reflexión que, desde el cuidado, nos lleve a abrir un debate más amplio donde, considero, se inserta de lleno la cuestión. Es así que, para tal fin, transitaremos por diversos conceptos, tales como: paradigma, ciencia, modernización reflexiva, medicina científicooccidental, física cuántica, etc.

Palabras clave: Enfermería, cuidado, paradigma, ciencia, modernización reflexiva, medicina científico-occidental. 


\section{INTRODUCCIÓN}

La enfermería siempre ha confiado en la importancia del cuidado humanizado. Y ello no es baladí, sobre todo, en los momentos donde el enfoque biologicista de la medicina científico-occidental ha llegado a ser casi insultante. En estas líneas propongo una reflexión que, desde el cuidado, nos lleve a abrir un debate más amplio donde, considero, se inserta de lleno la cuestión. Es así que, para tal fin, transitaremos por diversos conceptos, tales como: paradigma, ciencia, modernización reflexiva, medicina científico-occidental, física cuántica, etc.

\section{Desde el cuidado hasta el paradigma y la ciencia}

En la breve presentación de este texto, ya apostillé, la influencia que la Medicina convencional ejerce en la Enfermería. Siguiendo a Francine Saillant (2009) considerar que la medicina moderna -o mejor, científico-occidental (Perdiguero, 2009)- está caracterizada por una dicotomía entre dos espacios: el tratamiento técnico (cure) y los cuidados y los aspectos relacionales (care). Las peticiones, tanto desde dentro como desde fuera del espacio médico, hacia la humanización de la medicina, nos ponen en la pista de cómo, actualmente, en nuestro medio, la balanza está meridianamente posicionada hacia el cure y mucho menos hacia el care. Siendo así, también indiqué, supra, la sempiterna apuesta de la Enfermería por el cuidado humanizado -care con caring (ver abajo)-. De hecho -y de la mano, ahora también, de la revisión que Saillant (Ibíd.) hace de la temática en la bibliografía norteamericana- desde el otro lado del Atlántico, por ejemplo, tiempo atrás ya se sugiere truncar la relación, a saber, privilegiar el care frente al cure-; y todavía más, incluso se plantea la disociación absoluta entre ambas, lo que supondría separar la medicina de los cuidados. Asimismo, para trascender tal dicotomía y centrándose en el care, se propone el concepto de caring. Término tan complejo -aunque fundamentalmente sugiere proporcionar un reconocimiento positivo de carecomo su objeto -dar a la humanización una encarnación; la espiritualidad, la trascendencia y la estética...-. A la vez esta expresión, sugiere, la importancia que alcanza el vínculo social en el espacio terapéutico. Será pues el caring, el alma del care. Su carencia conduciría al cure, de ahí la importan- cia de visibilizarlo. En definitiva, tal como se aprecia, la Enfermería no elude el debate y muestra sin ambages y de manera comprometida, su posicionamiento y dirección al respecto.

Así las cosas, me interesa echar una mirada en nuestra "reciente" historia -y tal bisoñez es subrayable-, para que se pueda entender un poco mejor -al menos esa es la intención-, el porqué plantearnos, cada vez más abiertamente, el profundo calado del papel del cuidado -el care con caring- en el continuum salud/enfermedad. Creo acertado empezar por rastrear cuál es el paradigma que sustenta la ciencia en Occidente (Rico, 2009); para después, otear cómo ha mirado la medicina científico-occidental la enfermedad; y acercarnos, finalmente, a la moderna teoría de la física cuántica y su vinculación con el caring. Lo primero, nos lleva a recordar la propuesta que Thomas Kuhn (2004) en su obra de 1962 «La estructura de las revoluciones científicas»- hace de paradigma. Para él, es un modelo o patrón aceptado por una comunidad de científicos que raramente concurren en desacuerdo con su manera específica de hacer ciencia. Este modelo o patrón regirá la ciencia normal en un determinado periodo histórico, hasta su crisis y revolución. Las crisis y rupturas implicarían cambios radicales en la concepción de mundo, a las que este autor denomina «revoluciones científicas. En este sentido, como sugiere Stanislav Grof (2006), la influencia de los paradigmas no es sólo cognoscitiva, sino normativa, además de definir la naturaleza y la realidad, determina también el campo problemático permisible, los métodos de enfoque aceptables y establece los niveles de soluciones, hasta el punto que ciertos problemas hasta entonces cruciales pueden considerarse desatinados o acientíficos y algunos quedar relegados a otra disciplina. La ciencia normal basada en un nuevo paradigma no es sólo incompatible, sino inconmensurable con relación a la práctica regida por el anterior. Un buen ejemplo de la guía/limitación que describen los paradigmas, me parece, la reflexión hecha por Roger Bartra (2001), cuando analizó cómo las ciencias médicas del Renacimiento y los albores de la modernidad intentaban comprender el funcionamiento del cerebro humano: Los médicos de aquella época, dice Bartra, creían firmemente en las teorías humorales hipocráticas y galénicas, y por ello transitaban con 
facilidad del micromundo corporal al macrocosmos astronómico, atravesando ágilmente los mundos de la geografía, las costumbres, las estaciones, la alimentación y las edades. Entiendo, como Bartra, que rastrear el pasado nos ayuda a posicionar nuestro presente.

Definido el concepto y visto el calado de su expresión, aproximémonos a las bases del paradigma de la ciencia occidental. Desde aquí, se puede entender mejor el modus operandi de nuestra medicina. Dos son las figuras que construyen, con su pensamiento, la plataforma del modelo de la ciencia en nuestro medio, a saber: el filósofo, científico y matemático francés, René Descartes (1596-1650); y el matemático y físico británico, Isaac Newton (1642-1727). El primero aporta al paradigma, el conocido como dualismo cartesiano. En su obra de 1641, «Meditaciones metafísicas», presenta la mente y el cuerpo como dos sustancias diferentes, señalando la oposición irreconciliable entre sustancia pensante (inteligencia) y sustancia extensa (materia). Sea suficiente lo que recoge en la sexta meditación, para entender su planteamiento: como un reloj compuesto por ruedas y contrapesos (...) considero el cuerpo del hombre. A partir de aquí se deduce que la parte mecánica (cuerpo), puede ser separada, estudiada y reparada aisladamente. En realidad este posicionamiento deriva de las especulaciones filosóficas expuestas en su trabajo más conocido, «El discurso del Método» de 1637, donde abandonó los enfoques de la escolástica medieval. Desde el cógito, ergo sunt (pienso, luego existo) busca un nuevo método para, a través de la duda, hallar la única verdad cierta. Es entonces cuando plantea la visión mecanicista de la naturaleza, y propone la física y las matemáticas como medios de conocimiento del mundo material. La escisión mente/cuerpo también supone el origen del reduccionismo en Medicina, que, como explica Javier Granero (2003), se trata de la tendencia a explicar los fenómenos complejos en términos de componentes más simples.

Planteadas por Descartes las nuevas claves para interpretar el universo, Newton, por su parte, describe su funcionamiento mecánico. Defiende la teoría de la gravitación universal, basada en una fuerza que afecta a todos los cuerpos terrestres y espaciales. Además, describe el universo formado por materia sólida, cuyos elementos esenciales - los átomos- son indestructibles e inmutables. En el universo newtoniano las masas se atraen recíprocamente con una fuerza -la gravedad- que varía inversamente al cuadrado de la distancia existente entre ambas. Otro aspecto fundamental de su planteamiento es, desde la influencia de la geometría de Euclides, la concepción de un espacio tridimensional donde el tiempo actúa como un absoluto independiente, en relación a la materia. Como apunta Grof (Ibíd.), a propósito de este enfoque, la imagen resultante del universo es la de un gigantesco y perfectamente determinista mecanismo de relojería. El movimiento de la naturaleza obedece a leyes eternas e inmutables, y tanto los sucesos como los procesos en el mundo material consisten en cadenas interdependientes de causas y efectos.

La brillantez teórica de lo que se ha venido a denominar el paradigma newtoniano-cartesiano, ha hecho que su influjo llegue hasta hoy. Como hemos visto es a través del desarrollo de la física y las matemáticas, cuando se defiende que los fenómenos de la naturaleza están regulados por leyes mecánicas de la física y la química, y no por una inteligencia divina o supranatural. Por ello, este paradigma, también se conoce como modelo mecanicista. Y es que, como apunta David Le Bretón (1995), la inteligibilidad mecanicista convierte a las matemáticas en la clave única de comprensión de la naturaleza (...) el universo que se vive y que se siente tal y como aparece, gracias a las actividades perceptivas, cae en desgracia a favor de un mundo inteligible, puramente conceptual. Del mismo modo que la imaginación, los sentidos son engañosos, no podríamos basar en ellos la menor certeza racional. Las verdades de la naturaleza dejan de ser accesibles a la evidencia sensorial, hay que distanciarlas, purificarlas, someterlas a un cálculo racional. Entonces, la intensa adscripción al paradigma tiene sentido. Sobre todo, por su aplicabilidad a distintos planos de lo cotidiano. Ello es, en buena parte, lo que le ha conferido el marchamo de científico. Y éste, trasciende el campo de la física, ya que se ha hecho extensible a otras disciplinas como la Biología, o la propia Medicina, sin descartar a otras materias que se encuentran fuera de la órbita de las Ciencias Naturales, como la Sociología o la Antropología. En resumidas cuentas, cualquier disciplina que opera fuera del paradigma newtoniano-cartesiano, tiene un alto riesgo 
de no ser considerada científica.

En este punto conviene preguntarnos por la ciencia, es decir, hacer un esfuerzo de ejercicio epistemológico. Sin duda, la anterior puesta en escena de la conceptuación de paradigma, nos va ser de gran ayuda. Tomemos de partida, por ejemplo, la reflexión que sobre la ciencia hace Gregory Bateson (2002): la ciencia, como el arte, la religión, el comercio, la guerra y hasta el dormir, se basa en presupuestos. (...) en este empeño, es a todas luces deseable (pero no absolutamente necesario) que el científico conozca a conciencia sus propios presupuestos y sea capaz de enunciarlos. Es también conveniente y necesario para el discernimiento científico conocer los presupuestos de los colegas que trabajan en el mismo campo. Y sobre todo, es necesario que el lector de artículos científicos conozca los presupuestos de su autor. Siguiendo a Bateson, lo que me parece más interesante en este momento, es sondear la conciencia de los supuestos previos -pre-supuestos- que a la ciencia son de suyo. Y más en concreto, en la medicina científico-occidental. Me valgo para ello, ahora, del análisis de la Antropología Médica, fundamentalmente de un reciente trabajo de Ángel Martínez Hernáez (2008). Este autor, cuando sondea el tratamiento que la Antropología ha realizado sobre los temas de enfermedad y de los distintos sistemas médicos, lo hace a partir de la dialéctica entre lo racional y lo creencial. Por lo que aquí interesa, destacar que los antropólogos ocupados en este particular han sufrido una manifiesta evolución. De tal manera que, si inicialmente -Modelo Clásico- el abordaje de la enfermedad es marginal en la disciplina, y después -Modelo Pragmático- se evidencia una clara dependencia del paradigma de la medicina Occidental, en la actualidad -Modelo Crítico- se produce una difuminación de las fronteras entre ciencia y creencia, entre medicina y cultura. Y es aquí donde quería llegar, porque en este tercer estadio, la Antropología Médica, centra su interés en visualizar qué hay de creencial en la ciencia médica, y por extensión, cómo ésta construye sus objetos, desarrolla sus biotecnologías, genera sus identidades y representaciones culturales..., en conclusión, cómo la enfermedad no puede escapar de pre-supuestos que se construyen en un marco social, cultural, económico y político determinado. Lo destacable es que -Mishler citado por
Martínez Hernáez, Ibíd.- se considere a la biomedicina como una subcultura con sus creencias institucionalizadas, valores y prácticas $\mathrm{y}$, por tanto, susceptible de ser estudiada como otras instituciones culturales y sociales.

El ejercicio que la Antropología Médica está haciendo en este particular, me parece crucial cuando, como es aquí el caso, vamos buscando sondear lo científico/creencial en nuestra medicina. Pero cuidado, tal cometido, no supone caer en cierto grado de relativismo. Pues si bien, la intención es la contraria. Coincido, de hecho, con Alan Chalmer (1999) en que una cosa es impedir los usos ilícitos de las categorías de ciencia y método científico, y otra, apostar por las reacciones individualistas o relativistas de la ciencia. En este sentido, no se trata de que un punto de vista sea tan bueno como cualquier otro, porque si se quiere cambiar una situación de forma controlada, como mejor se logrará será comprendiendo la situación y dominando los medios disponibles para cambiarla. Entonces, la clave parece estar en poner todo el peso en la validación del conocimiento científico, sin perder de vista -como subrayamos atrás- la influencia de las circunstancias históricas, psicológicas y socio-culturales dadas. De hecho, como apostilla Beltrán Villalva (1999), que se cuestione el privilegio epistemológico atribuido al conocimiento científico no ha de llevar a considerarlo como una mera extensión del sentido común. Llegados aquí, entiendo de utilidad para establecer un marco de referencia que nos ubique respecto a la situación por la que está transitando la ciencia, revisar el distingo que expone Ulrich Beck (1998) entre la cientificación simple y la cientificación reflexiva. La primera hace referencia a la conceptualización, bajo criterios científicos, de amplios aspectos de la existencia humana; mientras que la segunda, es una fase de la cientificación, en la cual, ya no es sólo la naturaleza, el hombre y la sociedad lo que se somete a criterios científicos sino también la propia ciencia, sus productos, consecuencias y defectos. En esta etapa se produce una desmonopolización del conocimiento, donde no es el fracaso de las ciencias, sino su éxito, lo que las ha destronado, provocando una relativización de sus originarias pretensiones de validez. Para Ulrich Beck (2008) la cientificación reflexiva se inscribe como parte de lo que acontece en la 
radicalización de la modernidad, que trasciende la simple modernidad. Es lo que llama teoría de la modernización reflexiva.

\section{La medicina occidental y su relación con la ciencia}

En este momento no escapa al lector que, de esta "novedosa" realidad, no se libra nuestra medicina. Recuérdese -aunque sea de forma sucinta-, cómo, ésta, describe un particular modelo biologicista (Martínez Hernáez, Ibíd.), cuando, tras abandonar a lo largo del siglo XIX la nosotaxia more botanico, abraza el método anatomoclínico, es decir, apuesta por la incursión en el espacio de los órganos. Después, a fines de del XIX y principios del XX, se consolida el paradigma bacteriológico o teoría microbiana por el que, a una enfermedad concreta, le corresponde un determinado microorganismo patógeno y viceversa. Se demuestra así, la correspondencia entre el germen externo monomórfico y la enfermedad. Siguiendo su decurso, es durante el siglo XX hasta la actualidad, cuando se irá imponiendo la biología molecular. En esta nueva mirada el modelo biomédico pone en el punto de mira del origen de las enfermedades, la codificación celular; y de su tratamiento, la ingeniería genética. Atrás se anotó que, la medicina científico-occidental, se sustenta sobre el paradigma newtoniano-cartesiano. Un paradigma científico que como sabemos superó -o como diría Popper, falsó- a la física aristotélica. La teoría de Newton, como subraya Chalmers (Ibíd.), podía explicar la caída de los objetos y el funcionamiento de los sifones y bombas de extracción y podía también explicar los fenómenos que resultaban problemáticos para los aristotélicos. Además, la teoría de Newton podía explicar fenómenos a los que la teoría de Aristóteles no aludía, tales como las correlaciones entre las mareas y la posición de la luna, y la variación en la fuerza de la gravedad con la altura por encima del nivel del mar. Es por todo esto, que su poder explicativo la dote de gran éxito, y la medicina, como otras disciplinas científicas, se apoyen en ella. Pero, así las cosas, no se puede obviar que la propia ciencia en Occidente está descollando dicho arquetipo. Me refiero a que los físicos actuales explican la realidad desde la física cuántica, es decir, se trasciende la realidad entendida como lineal -mecanicista-, para llegar a otra llena de campos energéticos. Somos materia y energía, o mejor, como demostró Einsteim en su famosa ecuación (la Energía es igual a la Materia, multiplicada por la velocidad de la luz al cuadrado), ambas son la misma cosa. Lo que hace que esta ciencia de las posibilidades -cuántica- no describa el universo forjado por materia sólida, ni considere a los átomos -"elementos esenciales"- indestructibles e inmutables. Como sugiere Beltrán Villalva (Ibíd.), la mecánica cuántica introduce en la física un componente explosivamente no canónico. De tal manera que, si la teoría de Newton falsó la física aristotélica, la teoría de la relatividad de Einsteim -como también apostilla Chalmers (Ibíd.)- fue capaz de explicar los fenómenos que falsaron la teoría de Newton, al tiempo que era capaz de competir con la teoría newtoniana en las áreas en las que ésta había triunfado. Siendo así, podemos decir que Newton queda superado por Einsteim, o dicho de otro modo, si realmente nos posicionamos del lado de la ciencia, los referentes teóricos que sustentan nuestra medicina necesitan de una profunda revisión. Por cierto, hemos de añadir en relación a la debilidad científica del paradigma newtoniano-cartesiano, que desde que Benoit Mandelbrot propuso en 1975 la geometría fractal, su teoría permite aplicarse mucho mejor a una naturaleza compleja, irregular..., que era tarea imposible para la geometría tridimensional euclidiana de cubos, esferas y conos.

Sé que el asunto es controvertido y que son muchos los que reniegan de aplicar los conceptos de la física microscópica en el mundo macroscópico. Si bien, el error no es tanto negar el valor de cada física en su marco de acción, como su falta de integración. Bruce Lipton (2010) lo ilustra con el siguiente ejemplo: una enfermedad como el cáncer puede manifestarse a nivel superior cuando se siente o se ve el tumor. No obstante, el proceso que provocó el cáncer se inició a nivel molecular, dentro de las células progenitoras afectadas. De hecho, la mayor parte de las disfunciones biológicas (salvo las heridas causadas por traumatismos físicos) comienzan a nivel iónico y molecular en el interior de las células. Y éste es el quid de la cuestión de este texto, la física cuántica -o mundo cuántico invisible einsteiniano- puede ser una buena piedra de toque para indagar aspectos -antes insondables desde la óptica del mundo físico de Newton-, como la poderosa relación cuerpo- 


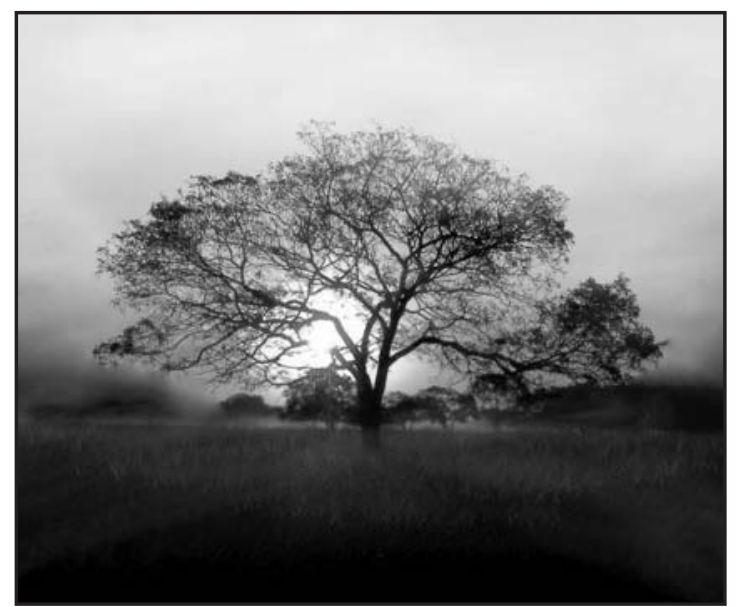

mente. Como apunta también el mismo autor (Ibíd.), la ciencia médica no deja de avanzar, pero los organismos vivos se niegan testarudamente a dejarse cuantificar. Las curaciones espontáneas, los fenómenos psíquicos, las asombrosas demostraciones de fuerza y resistencia, la habilidad para caminar sobre las brasas de un fuego sin quemarse, la capacidad de la acupuntura para disminuir el dolor mediante la canalización del chi a lo largo del cuerpo y otros muchos fenómenos paranormales desafían la biología newtoniana. Por cierto, quepa aquí inscribir los resultados expuestos en la tesis doctoral defendida recientemente por la enfermera María Teresa Pinedo -de la que se hace eco esta revista: Cultura de los Cuidados, II semestre 2009, $\mathrm{n}^{\mathrm{o}}$ 26-, donde se destacan los beneficios de las romerías ilicitanas para confrontar las enfermedades, concluyendo que: la fe tiene un efecto energético en las personas creyentes que les infunde fuerza para luchar contra un problema de salud; que, las convicciones religiosas son utilizadas como herramienta para una descarga emocional; o que, en los momentos de un diagnóstico nefasto, frente al dolor y la confusión inicial sentida, la persona actúa refugiándose en sus creencias como herramienta de consuelo y de esperanza que le anime a encontrar una salida.

Teresa Versyp (2005), en la presentación de la tercera edición de su conocido trabajo «La dimensión cuántica. De la física cuántica a la conciencia», señala que el mundo cuántico representa el campo de interconexión entre la energía y la materia, y es donde la complementariedad de la materia y de la conciencia, del cuerpo y de la mente, se vuelve más tangible. En tales dominios, la frontera entre ambas polaridades se vuelve difusa. Con la inmersión en la dimensión cuántica nuestra perspectiva se ensancha y abarcamos un poquito más. Los muros de nuestra lógica habitual se derrumban, el espacio tridimensional da paso a una realidad multidimensional. Entonces, como suscribe Larry Dossey (2006), la nueva perspectiva sobre la conciencia afirma $\sin$ ambages que la actividad mental consciente es capaz de producir efectos mensurables en el mundo físico, mundo en el que se incluyen el cuerpo, los órganos, los tejidos y las células del ser humano. La mente se ha convertido en un factor legítimo, real, del desarrollo de la salud y de la enfermedad. En la misma línea argumentativa Lipton (Ibíd.) espeta que, saber que son unos mecanismos tan distintos los que controlan la estructura y el funcionamiento de la materia debería haber ofrecido a la biomedicina nuevas expectativas de lo que son la salud y la enfermedad. Sin embargo, aún después de los descubrimientos de la física cuántica, a los estudiantes de medicina y de biología se les sigue enseñando a ver el cuerpo como una máquina física que opera según los principios de Newton. Debido a los prejuicios materialistas newtonianos, los investigadores convencionales han ignorado por completo el papel que juega la energía en la salud y en la enfermedad -señalar algunas excepciones, ya sea en pro de la mejora del diagnóstico (Tomografía Axial Computerizada o TAC, Imagen por Resonancia Magnética o RNM, Tomografía por Emisión de Positrones o TEP) o del tratamiento (litiasis renal tratada por ondas vibratorias)-. Y no pensemos que la cuestión en exceso novedosa, ya en 1974, McClare -citado en Lipton (Ibíd)-, un biofísico de la universidad de Oxford publicó un trabajo denominado «La resonancia en bioenergética». Allí revelaba que los mecanismos de las señales energéticas tales como las frecuencias electromagnéticas son cien veces más eficaces a la hora de transmitir información del entorno que las señales físicas como las hormonas, los neurotransmisores, los factores de crecimiento, etc. Desgraciadamente, tal como recogí del trabajo de Martínez Hernáez (Ibíd.), actualmente el modelo biomédico tiene puesta su mirada en la codificación celular y la ingeniería genética, despreciando totalmente el enfoque de las novedosas aportaciones de la física cuántica. 


\section{CONCLUSIÓN}

Aunque la cuestión no es nimia, de ahí que haya reservado la apostilla para la parte final del texto, no ha sido la intención de estas líneas sondear los frentes de resistencia a la integración -en pos de la ciencia-, del paradigma cuántico en la medicina occidental. Lo que interesa es subrayar que estamos en un momento de puesta en juicio del paradigma que nos ha servido, como expresaría Kuhn, de modelo o patrón aceptado por la comunidad de científicos que raramente concurren en desacuerdo con su manera específica de hacer ciencia. Como he señalado, el paradigma newtoniano-cartesiano, deja muchas lagunas sin resolver cuyo abordaje es factible desde los nuevos modelos físico-matemáticos, o dicho con otras palabras, desde la ciencia más actual. Y llegados a este punto, recojo el testigo de lo que expuse en la primera parte del artículo. Ya que, el salto cualitativo, se produce cuando estamos en el camino de la "demostración" científica del valor del caring en los procesos de curación y mantenimiento de la salud. Es así que cuando Saillant (Ibíd.) trataba con tino- su compleja implementación -en concreto, lo refería como idealización y utopía terapéutica- en la era de la reorganización de los sistemas sanitarios, no considerara el potencial futuro que puede ofrecernos el nuevo paradigma. Hasta ahora sólo hemos podido medir el cure, lo que explica el desequilibrio a su favor respecto al care. Las cosas pueden empezar a cambiar cuando las ciencias acreditadas den cabida, paradógicamente -siendo ciencia actualizada-, a las que están por acreditar. En ese camino, cada vez será menos esotérico afirmar que tu mente cambia tu biología, si como demuestra la nueva biología -Lipton (Ibíd.)- la energía de los pensamientos puede activar la producción de proteínas en la célula, es decir, impulsar la llave de la vida. De esta manera, el efecto placebo, la acción positiva de muchas de las terapias alternativas, el recurso a la fe, y por supuesto -por lo que aquí nos interesa-, el care con caring irán dejando el camino de la excepcionalidad en una ciencia que no puede explicar su papel sanativo-curativo. Será entonces, cuando empiecen a ocupar el rol que merecen. Aunque aún hemos de tener paciencia para llegar al escenario descrito pero sin marcha atrás, en un tiempo donde se impone la cientificación reflexiva-, me atrevo a afirmar que, Enfermería, desde siempre y $\sin$ dudarlo, ha apostado -sin ser nada fácil- por el caballo ganador: humanizar la atención sanitaria que presta.

\section{BIBLIOGRAFÍA}

- Bartra, R. (2001) Cultura y melancolía. Las enfermedades del alma en la España del Siglo de Oro. Anagrama, Barcelona.

- Bateson, G. (2002) Espíritu y naturaleza. Amorrortu, Buenos Aires.

- Beck, U. (1998) La sociedad del riesgo. Hacia una nueva modernidad. Paidós, Barcelona.

- Beck, U. (2008). La sociedad de riesgo mundial. En busca de la seguridad perdida. Paidós, Barcelona.

- Beltrán Villalva, M. (1999) Sobre la confianza en una ciencia secularizada. En Globalización, riesgo y reflexividad; tres temas de la teoría social contemporánea (García, F. y Ramos, R. ed.), CIS, Madrid.

- Chalmer, A. (1999) ¿Qué es esa cosa llamada ciencia? Una valoración de la naturaleza y el estatuto de la ciencia y sus métodos. Siglo XXI, Madrid pp. 123-141.

- Dossey, L. (2006) Tiempo espacio y medicina. Kairós, Barcelona.

- Grof, S. (2006) Psicología transpersonal. Nacimiento, muerte y trascendencia en psicoterapia. Kairós, Barcelona.

- Granero Liberta, J. (2003) Diferencias en los conceptos de salud y enfermedad en la medicina oficial y en las no convencionales, HUMANITAS. Humanidades Médicas, vol 1, $\mathrm{n}^{\mathrm{o}} 2$, pp. 21-30.

- Kuhn, T. (2004) La estructura de las revoluciones científicas. Fondo de Cultura Económico, Buenos Aires.

- Le Bretón, D. (1995) Antropología del cuerpo y modernidad. Nueva Visión, Buenos Aires.

- Lipton, B.H. (2010) La biología de la creencia. La liberación del poder de la conciencia, la materia y los milagros. Palmyra, Madrid.

- Martínez Hernáez, A. (2008) Antropología Médica. Teorías de la cultura, el poder y la enfermedad. Anthropos, Barcelona.

- Perdiguero, E. (2009) Sobre el pluralismo asistencial. En Enfermería y Antropología. Padeceres, cuidadores y cuidados (Comelles, J.M et al ed). Icaria, Barcelona pp 113-137.

- Rico Becerra, J.I. (2009) El inmigrante "enfermo". Apuntes y reflexiones desde un trabajo antropológico. Isabor, Murcia.

- Saillant, F. (2009) Cuidados, deseos vinculares y utopías terapéuticas: un análisis del concepto de caring. En Enfermería y Antropología. Padeceres, cuidadores y cuidados (Comelles, J.M et al ed), Icaria, Barcelona pp 189-218.

- Versyp, T. (2005) La dimensión cuántica. De la física cuántica a la conciencia. Plaza. Barcelona. 